\title{
Expression of mTOR pathway proteins in human amniotic fluid stem cells
}

\author{
NICOL SIEGEL, ALESSANDRO VALLI, CHRISTIANE FUCHS, \\ MARGIT ROSNER and MARKUS HENGSTSCHLÄGER \\ Medical Genetics, Medical University of Vienna, Währinger Gürtel 18-20, A-1090 Vienna, Austria
}

Received January 16, 2009; Accepted February 24, 2009

DOI: 10.3892/ijmm_00000192

\begin{abstract}
The discovery of human amniotic fluid stem cells initiated a new and promising stem cell research field. These cells harbor a high proliferative capacity and the potential to differentiate into cells of all three embryonic germ layers. The facts that they do not form tumors in vivo and do not raise the ethical concerns associated with human embryonic stem cells support their role as an optimal tool to study the underlying molecular mechanisms of cell differentiation processes and of their deregulation in human genetic diseases. Deregulation of the protein kinase mammalian target of rapamycin (mTOR) pathway is a hallmark of a wide variety of human genetic diseases. Here we report the establishment of an amniotic fluid stem cell line. We analysed the endogenous expression of the mTOR pathway proteins tuberin, mTOR, raptor, rictor, sin1, mLST8, Akt and p70S6K in human amniotic fluid stem cells. In addition, we studied the endogenous activity of the kinase p70S6K, one of the major targets of the mTOR complex 1 kinase, by analysing the p70S6K T389 phosphorylation status. The activity of the Akt kinase, the major mTOR complex 2 target, was studied by analysing its phosphorylation at $\mathrm{S} 473$. In addition, the mTOR inhibitor rapamycin was found to affect the phosphorylation status of p70S6K in amniotic fluid stem cells. Taken together, we provide evidence that the mTOR pathway is fully active in human amniotic fluid stem cells. These data demonstrate that amniotic fluid stem cell lines can be used as new tools to study the molecular and cell biological consequences of natural occurring alterations of the mTOR pathway being responsible for a wide variety of different human genetic diseases.
\end{abstract}

\section{Introduction}

Much of the recent excitement surrounding human stem cells is connected with the hope that they can be used for cell

Correspondence to: Dr Markus Hengstschläger, Medical Genetics, Medical University of Vienna, Währinger Gürtel 18-20, A-1090 Vienna, Austria

E-mail: markus.hengstschlaeger@meduniwien.ac.at

Key words: amniotic fluid, stem cell, mTOR, Akt, tuberin, raptor, rictor, p70S6K therapies for a wide variety of human diseases. The interest in these cells is based on the fact that they can differentiate into different cell lineages. Accordingly, they are also an optimal model to study the underlying molecular mechanisms of early and late cell differentiation processes. Especially, stem cells can be used to answer the question how these processes are affected/deregulated in cells harboring natural occurring molecular alterations being causatively involved in the development of specific human genetic diseases $(1,2)$.

Embryonic stem cells are very potent in regard to their differentiation potential, but can only be derived from the inner cell mass during embryonic development via destroying the human embryo. The usage of these cells for research and therapy has been constrained by different ethical considerations as well as by the risk of malignant development of remaining undifferentiated embryonic stem cells after transplantation into the patient's body $(2,3)$.

It is assumed that both, the differentiation potential and the proliferative capacity, of adult stem cells are limited compared to embryonic stem cells. However, importantly, adult stem cells do not raise ethical concerns and harbor a low risk of tumor development. Accordingly, many different studies have been initiated to search for alternative human sources for adult stem cells harboring the potential to differentiate into specific lineages (3-5). The recent success on induction of pluripotency directly in somatic cells enables the establishment of patient-specific induced pluripotent stem cells. Such diseasespecific stem cells are optimal biological models to recapitulate normal and pathological differentiation processes and human tissue formation in vitro, thereby enabling disease investigation and drug development (6-8).

Despite the wide and well-established usage of amniotic fluid cells in routine genetic prenatal diagnosis, for a long time almost nothing was known about their origins and properties. However, since the first evidence that human amniotic fluid could contain stem cells in 2003 (9), observations, such as that amniotic fluid stem cells harbor a high proliferative capacity and multilineage differentiation potential, initiated wide interest in this specific stem cell research field (10-12). A variety of different stem cell populations have been described to exist in human amniotic fluid, including e.g. stem cells expressing CD90, CD105, CD73 or CD166, but being negative for CD45, CD34 and CD14, or neurogenic stem cells. How-ever, most of the recent studies focus on a specific type of human amniotic fluid stem cells, expressing the transcription factor Oct-4 and CD117 (c-Kit), both well known markers for 


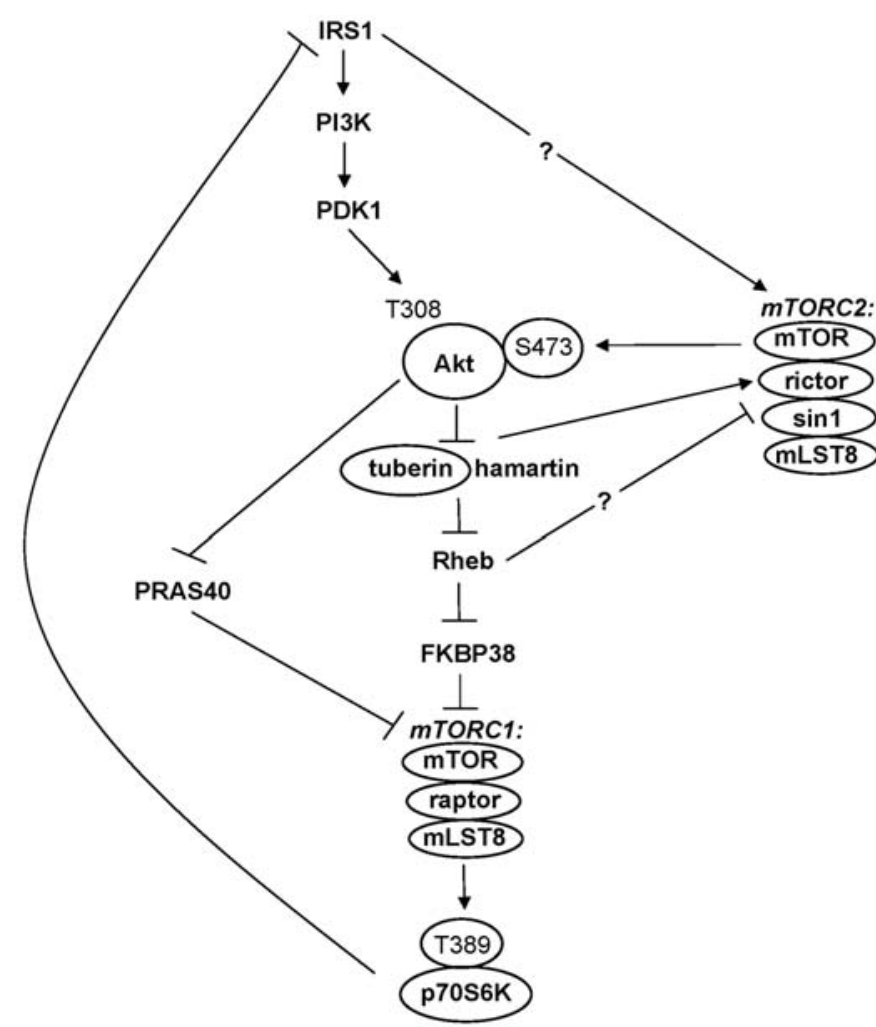

Figure 1. Schematic presentation of the mTOR pathway. The circled proteins and protein phosphorylation sites were investigated in this study. For details see the text.

pluripotency. Human amniotic fluid stem cells have been demonstrated to harbor high proliferation capacity and to not induce tumor formation in severe combined immunodeficient mice. These stem cells can be induced to differentiate along neurogenic, osteogenic, myogenic, endothelial, hepatic, and adipogenic lineages and they can develop and differentiate into de novo kidney structures during organogenesis in vitro (9,13-19). Taken together these fascinating findings make it tempting to speculate that human amniotic fluid stem cells might have advantages (such as a higher proliferation rate and a higher differentiation potential) over other adult stem cell types. Furthermore, they even have advantages (such as a lower risk for tumor development and no ethical concerns) over embryonic stem cells. Accordingly, human amniotic fluid stem cells are obviously an optimal biological system to study specific differentiation processes under normal and pathological conditions with the aim to clarify how the underlying molecular mechanisms are deregulated in cells harboring natural occurring molecular alterations being causatively involved in the development of specific human genetic diseases $(4,5,10,12,20,21)$.

The mTOR kinase plays a central role in the regulation of cell growth and cell proliferation. Two different mTOR containing complexes, named mTORC1 and mTORC2, have been identified in mammalian cells (Fig. 1). Besides mTOR protein, mTORC1 contains raptor (regulatory associated protein of mTOR), functioning as a scaffold for recruiting TORC1 substrates, and mLST8 (also known as GßL). The function of mLST8 is not really clarified. PRAS40 is phosphorylated by the oncogenic kinase Akt (also known as protein kinase B) releasing its inhibitory effects on mTORC1.
mTORC2 consists of the mTOR protein, mLST8, rictor (rapamycin-insensitive companion of mTOR) and sin1 (stressactivated protein kinase-interacting protein). The latter appear to stabilize each other through binding, building the structural foundation for mTORC2. mTORC1 is involved in the regulation of mRNA translation e.g. via its potential to phosphorylate and activate one of its major targets, the p70S6K (ribosomal p70S6 kinase), at T389 to activate the ribosomal protein S6. p70S6K also phosphorylates IRS1 promoting its degradation. mTORC2 phosphorylates Akt at S473, what in conjunction with the PDK1 (phosphoinositide-dependent kinase-1)-mediated phosphorylation at T308 drives full activation of Akt (22-24).

Upstream of mTOR activated receptor tyrosine kinases activate the phosphatidylinositol-3-kinase (PI3K) via e.g. the insulin receptor substrate 1 (IRS1). Phosphorylation of the membrane lipid phosphatidylinositol-4,5-biphosphate $\left(\mathrm{PIP}_{2}\right)$ by $\mathrm{PI} 3 \mathrm{~K}$ produces the second messenger phosphatidylinositol3,4,5-triphosphate $\left(\mathrm{PIP}_{3}\right)$. PDK1 and Akt bind to $\mathrm{PIP}_{3}$ at the plasma membrane, and, as already mentioned above, PDK1 phosphorylates Akt at T308. Akt-mediated phosphorylation down-regulates the GTPase activating potential of tuberin, the gene product of the tuberous sclerosis gene 2 (TSC2), toward Rheb (Ras homolog enriched in brain), which is a potent regulator of mTOR through FKBP38. Rheb has a negative effect on mTORC2, but the underlying mechanism remains unclear. In addition, it was shown that the tuberin/hamartin complex can associate with and positively regulate mTORC2, but not mTORC1 (22-27).

A wide variety of human diseases are known to be caused via deregulation of mTOR pathway components. Evidence has been provided for a role of mTOR in Alzheimer's disease, cardiac hypertrophy, obesity, type 2 diabetes, cancer and specific human genetic diseases e.g. upstream of mTOR the key signalling molecules Wnt, TNF- $\alpha$ Ras, PI3K, Akt, and Rheb are known to be deregulated in many human cancers. Mutations in the mTOR pathway component genes TSC1, TSC2, LKB1, PTEN, VHL, NF1 and PKD1 trigger the development of the syndromes Tuberous sclerosis, Peutz-Jeghers syndrome, Cowden syndrome, Bannayan-Riley-Ruvalcaba syndrome, Lhermitte-Duclos disease, Proteus syndrome, von Hippel-Lindau disease, Neurofibromatosis type 1, and Polycystic kidney disease, respectively $(23,26-28)$. Accordingly, it is of high relevance to identify a human stem cell model, with high proliferation capacity and differentiation potential, which can be used to investigate the role of alterations of the mTOR pathway for differentiation processes and human tissue formation in vitro. In this report we provide strong evidence that human amniotic fluid stem cells fulfil these criteria, thereby enabling the investigation of mTOR-associated diseases and drug development.

\section{Materials and methods}

Establishment of an amniotic fluid stem cell line. Human amniotic fluid cell samples were obtained from amniocentesis all performed between the 17th and 22nd week of pregnancy for routine prenatal diagnosis. The indications for amniocentesis were advanced maternal age or prenatally detected ultrasound signs, such as e.g. an increased nuchal translucency. 
Table I. Antibodies used in Western blot analyses.

\begin{tabular}{lll}
\hline Akt S473 & Cell Signaling & Cat. Number 4058 \\
Akt & Cell Signaling & Cat. Number 9272 \\
tuberin & Santa Cruz Biotechnology & Cat. Number sc-892 \\
mTOR & Cell Signaling & Cat. Number 2972 \\
raptor & Bethyl Laboratories & Cat. Number A300-506A \\
mLST8 & Cell Signaling & Cat. Number 3274 \\
rictor & Bethyl Laboratories & Cat. Number A300-459A \\
sin1 & Bethyl Laboratories & Cat. Number A300-910A \\
p70S6K T389 & Cell Signaling & Cat. Number 9234 \\
p70S6K & Cell Signaling & Cat. Number 9202 \\
$\alpha$-tubulin & Calbiochem & Cat. Number CP06 \\
\hline
\end{tabular}

This project has been reviewed and approved by the Ethics Commission of the Medical University of Vienna, Austria (project number 036/2002) and each patient signed a written informed consent $(9,14)$. Isolation of human amniotic fluid stem cells expressing CD117 antigen was performed via magnetic cell sorting using the CD117 MicroBead Kit (Miltenyi Biotec 130-091-332). The amniotic fluid stem cell line used, named CD117/2, was established by cell cultivation of a positively selected and eluted cell fraction (compare also ref. 18). All used human amniotic fluid cells were cytogenetically analysed according to standard protocols. Chromosome banding was produced by means of a conventional 550-band trypsin-Giemsa analysis. A significant number of metaphases were analysed for numerical and structural chromosome aberrations (29).

Cells and cell culture. The clonal human amniotic fluid stem cell lines Q1 and CB3 were kindly provided by Professor Anthony Atala. They have been established via selection for CD117-positivity as described above (18). All human amniotic fluid stem cells were grown in $\alpha$-MEM minimal essential medium (Gibco, Invitrogen), containing 15\% ESFBS (PAA), $2.5 \mathrm{mM}$ L-glutamine and antibiotics $(30 \mathrm{mg} / \mathrm{l}$ penicillin, $50 \mathrm{mg} / 1$ streptomycin sulphate, Sigma), supplemented with $18 \%$ Chang B and 2\% Chang C (Irvine Scientific) at $37^{\circ} \mathrm{C}$ and $5 \% \mathrm{CO}_{2}$. The non-transformed nonimmortalized IMR-90 fibroblasts were obtained from the American Type Culture Collection (CCL-186) and were grown in Dulbecco's modified Eagle's medium (DMEM) at $4.5 \mathrm{~g} / \mathrm{l}$ glucose, supplemented with $10 \%$ calf serum and antibiotics (30 mg/l penicillin, $50 \mathrm{mg} / 1$ streptomycin sulphate) at $37^{\circ} \mathrm{C}$ and $5 \% \mathrm{CO}_{2}$ and were routinely screened for mycoplasma (30). To confirm logarithmic growth cells were harvested, stained with propidium iodide and cytofluorometrically analysed for DNA content (31). For long-term treatments involving the pharmacological inhibitor of mTOR cells were grown in medium as described above and rapamycin (purchased from Calbiochem) was added to the medium at a final concentration of $100 \mathrm{nM} 24 \mathrm{~h}$ before protein extraction (30).

Protein extraction. Total protein was extracted of logarithmically growing cells by physical disruption of cell membranes by repeated freeze and thaw cycles. Cells were washed with PBS and harvested by trypsin incubation. Pellets were lysed in buffer A containing $20 \mathrm{mM}$ HEPES, $\mathrm{pH} 7.9,0.4 \mathrm{M} \mathrm{NaCl}$, $2.5 \%$ glycerol, $1 \mathrm{mM}$ EDTA, $0.5 \mathrm{mM}$ DTT, $1 \mathrm{mM}$ PMSF, $0.5 \mathrm{mM} \mathrm{NaF}, 0.5 \mathrm{mM} \mathrm{Na}_{3} \mathrm{VO}_{4}$ supplemented with $2 \mu \mathrm{g} / \mathrm{ml}$ aprotinin, $2 \mu \mathrm{g} / \mathrm{ml}$ leupeptin, $0.3 \mu \mathrm{g} / \mathrm{ml}$ benzamidinchlorid, $10 \mu \mathrm{g} / \mathrm{ml}$ trypsin inhibitor by freezing and thawing. Supernatants were collected by centrifugation at $15,000 \mathrm{rpm}$ for $20 \mathrm{~min}$ at $4^{\circ} \mathrm{C}$ and stored at $-80^{\circ} \mathrm{C}(32)$.

Immunoblotting. Proteins were run on an SDS-polyacrylamide gel and transferred to nitrocellulose. Blots were stained with Ponceau-S to visualize the amount of loaded protein (30). The antibodies used for immunodetection are described in Table I. Rabbit polyclonal and monoclonal antibodies were detected using anti-rabbit IgG, a HRP-linked heavy and light chain antibody from goat (A120-101P, Bethyl Laboratories); mouse monoclonal antibodies were detected using anti-mouse $\mathrm{IgG}$, an HRP-linked heavy and light chain antibody from goat (A90-116P, Bethyl Laboratories). Signals were detected using the enhanced chemiluminescence method (Pierce).

\section{Results}

Since human amniotic fluid contains different types of stem cells (10-12) we first established a cell line of CD117 (cKit)-positive human amniotic fluid stem cells. We used immunoselection via magnetic cell sorting to isolate CD117positive cells from human amniotic fluid cell samples. As described in an earlier report (18), these cells can be expanded in culture as stable stem cell lines. The human amniotic fluid stem cell line was named CD117/2. Cytogenetic analyses revealed normal karyotypes without any numerical or structural aberrations for all cells used in this study. Repeated cytogenetic analyses demonstrated genomic stability of the amniotic fluid stem cell lines. Flow cytometric investigations of the DNA content further showed that the here established amniotic fluid stem cell line follows logarithmic growth without significant appearance of apoptosis studied via the detection of subG1 phase cells (data not shown). The cells grow with no need for feeder layers and without evidence for spontaneous differentiation. Freezing and thawing 


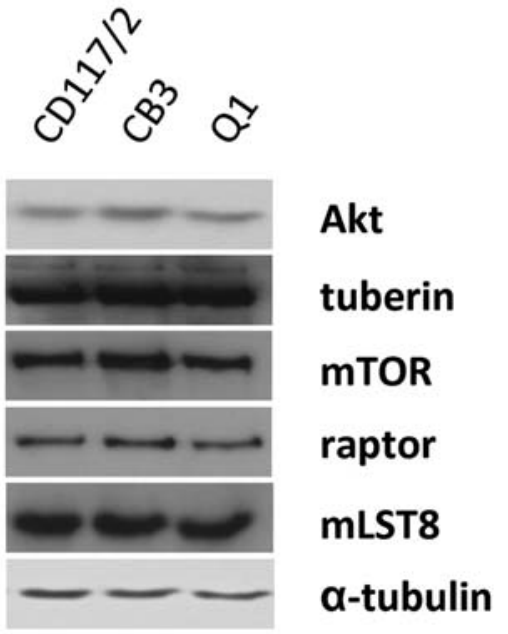

Figure 2. mTOR pathway protein levels in three different human amniotic fluid stem cell lines. Total protein of logarithmically growing CD117/2, CB3 and Q1 cells was extracted. Western blot analyses of Akt, tuberin, mTOR, raptor and mLST8 protein levels were performed (for details of the used antibodies see Table I). Detection of $\alpha$-tubulin was included to prove equal loading of lysates.

did not affect the cell growth properties of these cells. Concerning morphology and cell growth properties the cell line CD117/2 is very comparable to two other human amniotic fluid stem cells lines, named CB3 and Q1, which have earlier been isolated and established using the same approach (18).

Since alterations of the mTOR pathway are involved in a wide variety of different human genetic diseases $(23,26,27)$, it was the aim of this study to clarify whether the cell line CD117/2, CB3 and Q1, can be used as biological systems to study endogenous regulation of mTOR. As proven by cell doubling studies and flow cytometric DNA analyses (data not shown) the cells were growing logarithmically at the time point of protein extraction. After determination of the protein concentration using the Bio-Rad protein assay with bovine serum albumin as the standard, exactly the same amount of total protein of the three stem cell lines, CD117/2, CB3 and $\mathrm{Q} 1$, was run on an SDS-polyacrylamide gel and transferred to nitrocellulose. Blots were stained with Ponceau-S to visualize the amount of loaded protein. Ponceau-S staining proved that the amount of protein loaded on each lane was equal in the presented Western blot analysis. In addition, we co-analysed $\alpha$-tubulin expression, which is not involved in the mTOR pathway. Analysis of $\alpha$-tubulin further confirmed equal protein loading (Fig. 2). First, we studied the endogenous protein levels of mTOR, raptor and mLST8, the components of mTORC1 (Fig. 1). Western blot analyses revealed that in all three human amniotic fluid stem cell lines, all effectors of mTORC1, can be detected studying endogenous protein levels. In addition, the two major upstream regulators of mTORC1, Akt and tuberin, can also be investigated in these stem cell lines under the experimental conditions described, using the indicated antibodies (Table I, Fig. 2).

Besides mTORC1, another mTOR containing kinase complex, named mTORC2, has been identified in mammalian cells. mTORC2 consists of the mTOR protein, $\mathrm{mLST} 8$, rictor and $\sin 1$. The latter two proteins are specific for mTORC2

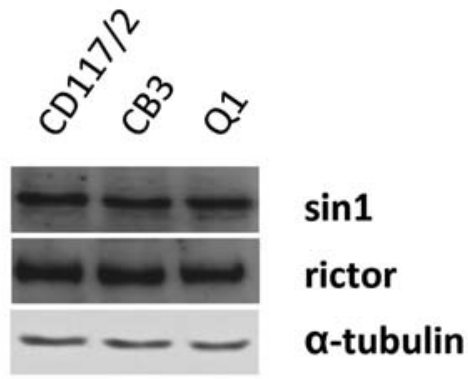

Figure 3. The levels of mTORC2-specific proteins in three different human amniotic fluid stem cell lines. Total protein of logarithmically growing CD117/2, CB3 and Q1 cells was extracted. Western blot analyses of sin1 and rictor protein levels were performed (for details of the used antibodies see Table I). Detection of $\alpha$-tubulin was included to prove equal loading of lysates.

(Fig. 1) (22-24). Western blot analyses revealed that all three human amniotic fluid stem cell lines express detectable endogenous levels also of $\sin 1$ and rictor (Fig. 3). The findings obtained so far provide evidence that human amniotic fluid stem cells can be used to study the mTOR containing complexes and the relevance of their alterations for cell differentiation processes. The fact that the three amniotic fluid stem cell lines, CD117/2, CB3 and Q1, express very comparable levels of endogenous Akt, tuberin, mTOR, raptor, rictor, and mLST8 provide additional evidence that they represent comparable cell populations. Taken together, these data suggest that an active mTOR pathway is a characteristic of human amniotic fluid stem cells. However, the observation that all mTOR complex components are expressed in human amniotic fluid stem cells does not necessarily mean that mTORC 1 and mTORC2 are active kinase complexes in these cells.

Both, mTORC1 and mTORC2 activity, are regulated on several levels, including complex formation, phosphorylation or intracellular localization (Fig. 1) $(23,26-28)$. The p70S6K is a major downstream target of mTORC1. mTORC1 phosphorylates and activates p70S6K at T389 to activate the ribosomal protein S6 via phosphorylation. Accordingly, analysing endogenous levels of phosphorylated p70S6K T389 as a functional readout is a widely used approach allowing conclusions on endogenous mTORC1 activity. Western blot analyses demonstrated that the p70S6K protein is expressed and phosphorylated at T389 in all three studied human amniotic fluid stem cell lines (Fig. 4). These data provide strong evidence that $\mathrm{mTORC} 1$ is active as a kinase complex in these cells. On the other hand, since the major target of mTORC2 is Akt (Fig. 1), analysing the phosphorylation status of Akt S473 is widely used as an endogenous functional readout of mTORC2 kinase activity. The detection of endogenous Akt protein and Akt S473 phosphorylation (Fig. 5) proves that also mTORC2 is active in these cells and that these stem cell lines can be used to study its regulation.

To ensure that human amniotic fluid stem cells exhibit normal regulation of the mTOR pathway we employed a widely used approach to study the effects of blocking mTOR kinase on a major mTOR substrate. As performed in many earlier studies, we made use of the detection of p70S6K protein migration shifting from the slower migrating phosphorylated form to the faster migrating hypophosphosphorylated 


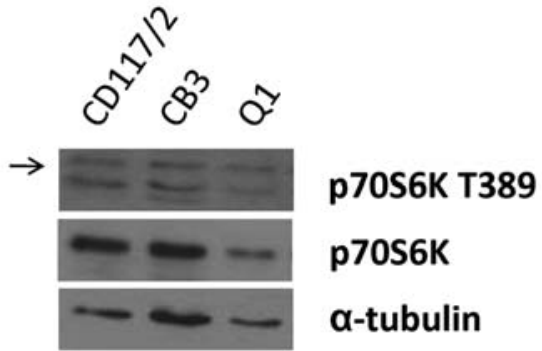

Figure 4. Endogenous p70S6K activity in three different human amniotic fluid stem cell lines. Total protein of logarithmically growing CD117/2, CB3 and Q1 cells was extracted. Western blot analyses of phosphorylated p70S6K T389 (arrow) and of total p70S6K protein levels were performed (for details of the used antibodies see Table I). Detection of $\alpha$-tubulin was included to prove equal loading of lysates.
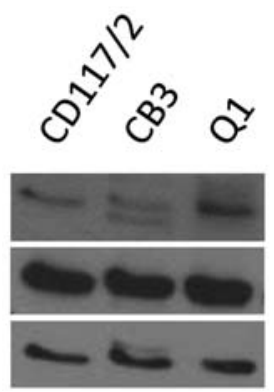

\section{Akt S473 \\ Akt \\ a-tubulin}

Figure 5. Endogenous Akt activity in three different human amniotic fluid stem cell lines. Total protein of logarithmically growing CD117/2, CB3 and Q1 cells was extracted. Western blot analyses of phosphorylated Akt S473 and of total Akt protein levels were performed (for details of the used antibodies see Table I). Detection of $\alpha$-tubulin was included to prove equal loading of lysates.

form upon treatment with an mTOR inhibitor. Downregulation of mTOR activity was triggered via treatment with the mTOR inhibitor rapamycin. Rapamycin bound to the protein FKBP12 generates a drug-receptor complex that binds and inhibits mTORC1. FKBP12-rapamycin suppresses the assembly of mTOR/raptor (compare e.g. refs. 30,33). Using this approach we observed the effects of rapamycin on p70S6K to be very comparable between human amniotic fluid stem cells and non-transformed non-immortalized primary human fibroblasts (IMR-90 cells) (Fig. 6). These data demonstrate that the mTOR pathway in human amniotic fluid stem cells is regulated and controlled as in normal human control cells.

\section{Discussion}

Deregulation of the mTOR pathway is a hallmark of a wide variety of human genetic diseases. The mTOR pathway has been demonstrated to be causatively involved in Alzheimer's disease, cardiac hypertrophy, obesity, type 2 diabetes, cancer and many specific human genetic diseases. Accordingly the establishment of biological tools to study the molecular and cell biological consequences of natural occurring alterations of the mTOR pathway being responsible for a wide variety of different human genetic diseases is of highest relevance $(23,26-28)$.

In the past, tumor cell lines or transformed derivatives of native tissues from diseased patients represented the most

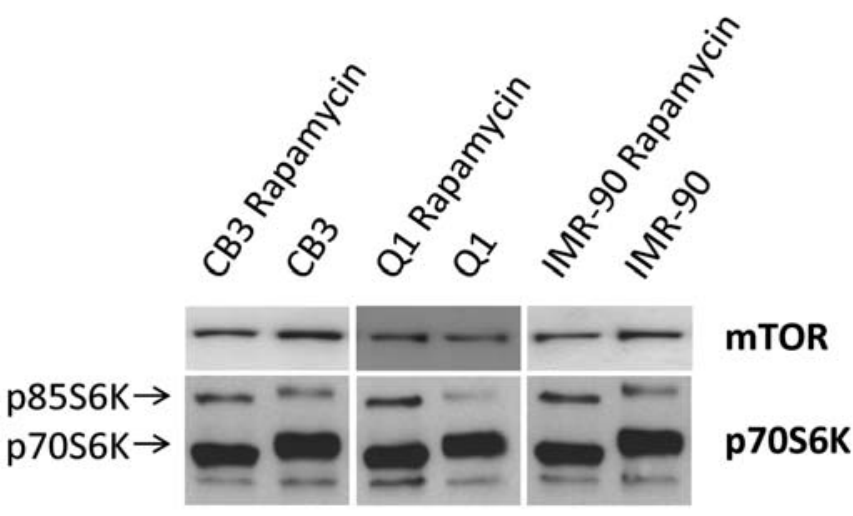

Figure 6 . The mTOR inhibitor rapamycin affects the p70S6K phosphorylation status in human amniotic fluid stem cells. Logarithmically growing amniotic fluid stem cells (CB3 and Q1 cells) and primary non-transformed nonimmortalized IMR-90 fibroblasts were treated with rapamycin $(100 \mathrm{nM})$ for $24 \mathrm{~h}$. Total lysates of so treated cells were analysed for p70S6K protein migration and for mTOR protein levels via Western blotting (for details of the used antibodies see Table I)

commonly used biological systems for such research studies. However, a very potent alternative approach has been established as a consequence of the recent success on induction of pluripotency directly in somatic cells. It has been demonstrated to be possible to establish patient-specific induced pluripotent stem cells. Such disease-specific stem cells, providing an optimal biological model to recapitulate normal and pathological differentiation processes in vitro, have e.g. been established from patients diagnosed with amyotrophic lateral sclerosis, adenosine deaminase deficiency-related severe combined immunodeficiency, Swachman-Bodian-Diamond syndrome, Gaucher disease type III, Duchenne and Becker muscular dystrophy, Parkinson disease, Huntington disease, type 1 diabetes mellitus and Down syndrome (6-8).

The syndromes Tuberous sclerosis, Peutz-Jeghers syndrome, Cowden syndrome, Bannayan-Riley-Ruvalcaba syndrome, Lhermitte-Duclos disease, Proteus syndrome, von Hippel-Lindau disease, Neurofibromatosis type 1, and Polycystic kidney disease are caused by mutations in the mTOR pathway component genes TSC1, TSC2, LKB1, PTEN, VHL, $N F 1$ and $P K D 1$, respectively $(23,26-28)$. It would be of enormous help for the research in this field to define a biological stem cell systems harboring natural occurring disease causing mutations in these genes. Establishing patient-specific induced pluripotent stem cells, as described above, would obviously be a very promising strategy.

Recently, we have suggested another approach; amniotic fluid stem cells harbor a high proliferative capacity and the potential to differentiate into cells of all three embryonic germlayers, they do not form tumors in vivo and they do not raise the ethical concerns associated with human embryonic stem cells. Compared with induced pluripotent stem cells amniotic fluid stem cells do not need to undergo prior processes of inducing stem cell properties. World-wide, amniocenteses are performed for prenatal genetic diagnosis of monogenetic diseases. We suggested it to be a promising strategy of highest value for basic research to establish clonal amniotic stem cell lines derived from a wide variety of pregnancies with specific genetic aberrations (10). 
However, to support the notion that such a strategy would be worthwhile two major questions must be answered: a) is the establishment of human amniotic fluid stem cell lines a reproducible approach? b) is it possible to study the mTOR pathway and its alterations on endogenous levels in human amniotic fluid stem cells? In this report we answered the first question by establishing a new CD117-positive human amniotic fluid stem cell line, with properties very comparable to those of two amniotic fluid stem cell lines established earlier (18) via the identical approach of magnetic cell sorting and cell cultivation. In addition, studying endogenous proteins we have proven the expression of the mTOR pathway components tuberin, mTOR, raptor, rictor, sin 1, mLST8, Akt and p70S6K in these three human amniotic fluid stem cell lines. Furthermore, we have demonstrated both, mTORC1 and mTORC2, to be active in amniotic fluid stem cells. By analysing the p70S6K T389 phosphorylation status we found the kinase p70S6K, one of the major targets of the mTOR complex 1 kinase, to be active. The activity of Akt kinase, the major mTOR complex 2 target, was proven by investigating its phosphorylation at S473. In addition, that human amniotic fluid stem cells exhibit normal regulation of the mTOR pathway was demonstrated by studying the effects of blocking mTOR kinase activity via its inhibitor rapamycin.

The findings presented in this study provide strong evidence that the mTOR pathway is fully active in human amniotic fluid stem cells and that this stem cell type can be used as a new model to study the molecular and cell biological consequences of naturally occurring alterations of the mTOR pathway, thereby enabling studies regarding the underlying mechanisms of a wide variety of human genetic disease with the aim to discover new therapeutic strategies.

\section{Acknowledgements}

We wish to thank Professor Anthony Atala for kindly providing the clonal human amniotic fluid stem cell lines Q1 and CB3. Research in our laboratory is supported by the FWF Austrian Science Fund (P18894-B12), by the Herzfelder'sche Familienstiftung and by the Research Training Network 'Developing a stem cell based therapy to replace nephrons lost through reflux nephropathy' (http://www.kidstem.org) funded by the European Community as part of the Framework program 6 (FP6 036097-2).

\section{References}

1. Rosenthal N: Prometheus's vulture and the stem-cell promise. N Engl J Med 349: 267-274, 2003.

2. Cedar SH, Cooke JA, Luo Z, et al: From embryos to embryonic stem cells: biopolitics and therapeutical potential. Reprod Biomed Online 13: 725-731, 2006

3. Pennings G: The ethics of using embryos in research. Reprod Biomed Online 14: 92-97, 2007.

4. Schulman A: The search for alternative sources of human pluripotent stem cells. Stem Cell Rev 1: 291-292, 2005.

5. Edwards RG and Hollands P: Will stem cells in cord blood, amniotic fluid, bone marrow and peripheral blood soon be unnecessary in transplantation? Reprod Biomed Online 14: 396-401, 2007.

6. Dimos JT, Rodolfa KT, Niakan KK, et al: Induced pluripotent stem cells generated from patients with ALS can be differentiated into motor neurons. Science 321: 1218-1221, 2008.
7. Park I-H, Arora N, Huo H, et al: Disease-specific induced pluripotent stem cells. Cell 134: 877-886, 2008.

8. Maherali N and Hochedlinger K: Induced pluripotency of mouse and human somatic cells. Cold Spring Harb Symp Quant Biol [Epub ahead of print], 2008.

9. Prusa AR, Marton E, Rosner M, et al: Oct4 expressing cells in human amniotic fluid: a new source for stem cell research? Hum Reprod 18: 1489-1493, 2003.

10. Siegel N, Rosner M, Hanneder M, et al: Stem cells in amniotic fluid as new tools to study human genetic diseases. Stem Cell Rev 3: 256-264, 2007.

11. Simantov R: Amniotic stem cell international. Reprod Biomed Online 16: 597-598, 2008

12. Aboushwareb T and Atala A: Stem cells in urology. Nat Clin Pract Urol 5: 621-631, 2008.

13. In t'Anker PS, Scherjon SA, Kleijburg-van der Keur C, et al: Amniotic fluid as a novel source of mesenchymal stem cells for therapeutic transplantation. Blood 102: 1548-1549, 2003.

14. Prusa AR, Marton E, Rosner M, et al: Neurogenic cells in human amniotic fluid. Am J Obstet Gynecol 191: 309-314, 2004

15. Tsai M-S, Lee J-L, Chang Y-J, et al: Isolation of human multipotent mesenchymal stem cells from second-trimester amniotic fluid using a novel two-stage culture protocol. Hum Reprod 19: 1450-1456, 2004

16. Karlmark KR, Freilinger A, Marton E, et al: Activation of ectopic Oct4 and Rex-1 promoters in human amniotic fluid cells. Int J Mol Med 16: 987-992, 2005.

17. Bossolasco P, Montemurro T, Cova L, et al: Molecular and phenotypic characterization of human amniotic fluid cells and their differentiation potential. Cell Res 16: 329-336, 2006.

18. De Coppi P, Bartsch G, Siddiqui MM, et al: Isolation of amniotic stem cell lines with potential for therapy. Nat Biotechnol 25: 100-106, 2007.

19. Perin L, Giuliani S, Jin D, et al: Renal differentiation of amniotic fluid stem cells. Cell Prolif 40: 936-948, 2007.

20. Murray P, Camussi G, Davies J, et al: The KIDSTEM European research training network. Developing a stem cell based therapy to replace nephrons lost through reflux nephropathy. Organogenesis 3: 1-4, 2007.

21. Perin L, Giuliani S, Sedrakyan S, et al: Stem cell and regenerative science applications in the development of bioengineering of renal tissue. Pediatr Res 63: 467-471, 2008.

22. Yang Q and Guan K-L: Expanding mTOR signaling. Cell Res 17: 666-681, 2007.

23. Dann SG, Selvaraj A, Thomas G, et al: mTOR Complex1-S6K1 signaling: at the crossroads of obesity, diabetes and cancer. Trends Mol Med 13: 252-259, 2007.

24. Bhaskar PT and Hay N: The two TORCs and Akt. Dev Cell 12: 487-502, 2007.

25. Wullschleger S, Loewith R, Hall MN, et al: TOR signaling in growth and metabolism. Cell 124: 471-484, 2006.

26. Guertin DA and Sabatini DM: Defining the role of mTOR in cancer. Cancer Cell 12: 9-22, 2007.

27. Rosner M, Hanneder M, Siegel N, et al: The mTOR pathway and its role in human genetic diseases. Mutat Res 659: 284-292, 2008.

28. Chiang GG and Abraham RT: Targeting the mTOR signaling network in cancer. Trends Mol Med 13: 433-442, 2007.

29. Hengstschläger M, Prusa A, Repa C, et al: Subtelomeric rearrangements as neutral genomic polymorphisms. Am J Med Genet 133: 48-52, 2005.

30. Rosner M and Hengstschläger M: Cytoplasmic and nuclear distribution of the protein complexes mTORC1 and mTORC2: rapamycin triggers dephosphorylation and delocalization of the mTORC2 components rictor and $\sin 1$. Hum Mol Genet 17: 2934-2948, 2008.

31. Rosner M, Hofer K, Kubista M, et al: Cell size regulation by the human TSC tumor suppressor proteins depends on PI3K and FKBP38. Oncogene 22: 4786-4798, 2003.

32. Rosner M, Freilinger A, Hanneder M, et al: p27 localization depends on the tumor suppressor protein tuberin. Hum Mol Genet 16: 1541-1556, 2007.

33. Sarbassov DD, Ali SM, Sengupta S, et al: Prolonged rapamycin treatment inhibits mTORC2 assembly and Akt/PKB. Mol Cell 22: 159-168, 2006. 